\title{
$\bullet$ Perspective of Ocular Manifestations in Coronavirus Infection: A Review
}

\section{IJCRR}

Section: Healthcare

ISI Impact Factor

(2019-20): 1.628

IC Value (2019): 90.81 $\operatorname{SJIF}(2020)=7.893$

(c) (i) (3)

Copyright@IJCRR

\section{Gupta $\mathbf{N}^{1}$, Nagpal $\mathbf{D}^{2}$, Panda $\mathbf{S N}^{2}$}

'Chitkara School of Health Sciences, Chitkara University, Punjab, India; ${ }^{2}$ Chitkara University lnstitute of Engineering \& Technology, Chitkara University, Punjab, India.

\section{ABSTRACT}

Coronavirus (COVID-19), the disease has become pandemic within a short span of time. Transmission of this disease occurs with the secretions from infected individuals to another directly. The secretions can be inhaling of droplets, saliva, or droplets of the infected person. It is a controversial statement if this pandemic can be transmitted through tears. However, this affects various parts of the body, and there exist associated ocular findings. Considering the reported studies on the ocular signs and symptoms of the disease, it has certainly drawn attention to the eye's anterior segment. These findings include conjunctivitis, epiphora, and chemosis. This narrative review aims to understand the impact of coronavirus on the retina as there are numerous families of viruses that contribute to retinopathy. This review focuses on the imaging techniques that can be implemented to assess the affected patients' retina. The pandemic has re-enforced health systems to evolve with technology; hence artificial intelligence can be considered to evaluate the ocular signs in patients who report with COVID-19.

Key Words: COVID-19, Coronavirus, Ocular symptoms, Ocular signs, Retina, Fundus imaging

\section{INTRODUCTION}

The Coronavirus (COVID-19) is a pandemic, which was generally evolved in Wuhan, China. The initial case was presented as pneumonia which eventually turned into a severe acute respiratory syndrome (SARS-CoV). The diagnosis of COVID 19 at the early stages is necessary to prevent the virus from dispersing from one person to another. It usually has four stages, namely imported cases, local transmission, community transmission, epidemic. Although this pandemic has reached stage four, hence early detection and appropriate treatment become mandatory.

The name Coronavirinae $(\mathrm{CoV})$ is derived from a Latin word corona means crown. The family Coronaviridae has a subfamily CoVs. These are further divided into four genera, namely alphacoronavirus, betacoronavirus, gamma coronavirus, and delta coronavirus. These zoonotic viruses that are regularly transmitted from animal species to humans. ${ }^{1}$ Among this alpha- or the beta- $\mathrm{CoV}$ is known to cause the disease among humans. The seven types of $\mathrm{CoVs}^{2}$ known to infect humans are illustrated in Figure 1.

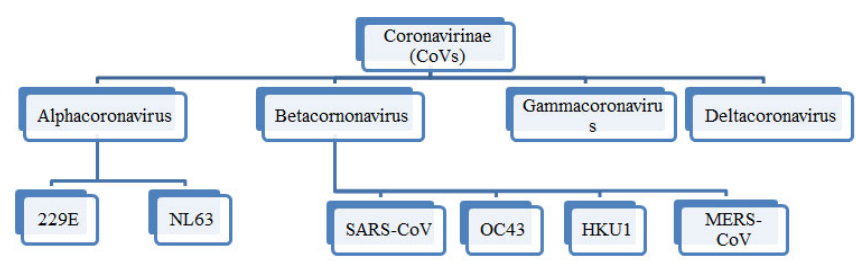

Figure 1: Types of Coronavirinae (CoVs).

The crown-shaped COVID-19 has an approximate diameter between 6 and $140 \mathrm{~nm}$ and is capped by 9 to $12 \mathrm{~nm}$ lengthened typical spikes. ${ }^{3}$ The virus is known to enter the human cell and binds with the angiotensin-converting enzyme (ACE) 2 protein. This protein is usually found to be present in the epithelial cells of the lungs and other tissues. Patients with diabetes mellitus and hypertension are found to have increased expression of ACE-2 protein. ${ }^{4}$

Transmission of the virus as postulated can be through contaminated dry surfaces or self-inoculation of mucous membranes of the nose, eyes, or mouth. ${ }^{5,6}$ The survival of viruses in the environment depends upon the temperature, nature of the surface, and relative humidity. It can survive more than

\section{Corresponding Author:}

Nagpal D, Chitkara University Institute of Engineering \& Technology, Chitkara University, Punjab, India.

Email: dimple.nagpal@chitkara.edu.in

ISSN: 2231-2196 (Print)

Received: 10.12 .2020
ISSN: 0975-5241 (Online)

Revised: 04.01 .2021
Accepted: 06.02.2021
Published: 30.03 .2021 
24 hours on cardboard and multiple days on plastic and other surfaces. In today's scenario, research in the field of coronavirus is at a higher pace. There are various methods to detect COVID 19 which majorly includes risk stratification, radiological techniques, however, there are many asymptomatic individuals.

This study aims to understand the ocular symptoms of COVID 19 amidst the eye professionals and propose the ocular manifestation in the retina as the pandemic's growth occurs. Initially, this new disease was reported by an ophthalmologist who observed various ocular secretions resulting in conjunctivitis or conjunctival congestion in such patients. The need for face-to-face proximity to examine the COVID-19 patient through slit lamps and ophthalmoscope ${ }^{7}$ may increase the chances of spreading the disease among eye care professionals or ophthalmologists. Several safety measures for ophthalmologists are taken to prevent transmission. As per the report, installing a protective shield on slit lamps was implemented to prevent transmitting the disease along with a surgical mask.

There are various preventive measures for ophthalmologists that should be taken care of during diagnosing such patients. The precautions include patient isolation, sanitization, wearing a mask, gloves, goggles, shield, gowns, silence, fast track, outdoor clothes, etc. ${ }^{8}$ However, the correlation of COVID 19 with any of the ocular manifestations is still in its onset, to uncover the disease's suspicion. ${ }^{9}$ In this paper, the ocular manifestations and corona virus-induced retinal changes are being reviewed.

\section{CLINICAL SIGNS AND SYMPTOMS}

The nature of COVID 19 patients can be symptomatic as well as asymptomatic. The various features observed in symptomatic COVID 19 patients can be respiratory or marginal. The common respiratory features include fever, headache, diarrhea, fatigue, cough, sputum production, and myalgia. ${ }^{10,11}$ The marginal symptoms include gastrointestinal inflammation, ocular congestion, skin rashes, headache, and loss of taste or smell, ${ }^{12}$ whereas shortness of breath, chest pain, loss of speech or movement are some of the serious symptoms.

Although there are various symptoms for the detection of COVID-19, the precise diagnosis of the disease is still elusive.

\section{OCULAR TRANSMISSION AND PATHOGENESIS}

The only source of transmission of the disease through the eyes is the tears, therefore it is alarming for ophthalmologists to diagnose COVID 19 in the eyes as well. However, the re- searchers explain its transmission and pathogenesis through ACE 2 protein as well. The presence of ACE- 2 is also seen in the anterior segment of the eye, which is similar to receptors of avian influenza species and the human influenza virus. These are extensively present in the corneal and conjunctival epithelium, similar to the nasal and tracheal mucosal lining. ${ }^{13,14}$ Hence it can be proposed that the transmission of this virus can occur through the tears in the ocular surface to the nasal cavity and tracheal tissues. However, the tears get renewed constantly by the lacrimal glands in the eye, and it can be speculated that the virus enters the tears in the form of droplets, which might be then transferred to the nasal and tracheal tissues.

Choudhary et al. in 2017 postulated that the ACE- 2 protein is majorly expressed in the posterior segment of the eye and can be commonly seen in the retinal pigment epithelium. ${ }^{15} \mathrm{~A}$ few studies suggest that COVID-19 is proficient in producing a wide spectrum of ocular manifestations including sightthreatening conditions such as retinitis and optic neuritis. The virus may develop in-vivo mutations that can transform the manifestations of the disease. At present, there is limited literature supporting the COVID-19 infection through ACE-2 and its adverse effects in the eye. ${ }^{16}$ As this pandemic continues, a better understanding of the virus is required to understand its pathogenesis in the eye.

\section{OCULAR MANIFESTATIONS OF COVID 19}

Studies report that $30 \%-40 \%$ of the patients presenting with positive COVID-19 manifest ocular findings which are consistent with conjunctivitis. ${ }^{17}$ This may also be accompanied by other ocular symptoms such as epiphora, conjunctival chemosis, and anterior uveitis. The potential sources of transmission are the mucous membrane of the eye. According to a recent update by the American Academy of Ophthalmologists (AAO), conjunctivitis can be one of the symptoms in patients having this disease. ${ }^{18}$ If left untreated, it can result in keratoconjunctivitis and ultimately lead to severe blindness. ${ }^{19}$ Studies suggested the presence of the virus on the ocular mucosa is $35 \%$ of the eye CoVs and $10 \%$ of the associated conjunctivitis cases. Tears contact may thus rapidly lead to the spread of the virus which is a contradictory. ${ }^{16,18}$

\section{Can COVID-19 affect the Retina?}

The COVID-19 pandemic has a massive impact on the human biological system, the associated coronaviridae decreases the cell-mediated immunity and makes the person highly susceptible to opportunistic viral ocular infections as well. Retinal findings in COVID 19 patients are still a mystery. It can be hypothesized that the pathogenesis of conjunctival and retinal microvasculopathy is perhaps similar and might involve increased plasma viscosity, circulating immune centers, and 
infectious injury of the vasculature. ${ }^{19}$ In the existing literature, there are many groups of viruses which have significant retinal manifestations such as the Paramyxoviridae family, Picornaviridae family and Herpesviridae family. ${ }^{20}$

Typically, the major group of viral ocular manifestations includes: opportunistic infections, neoplasms, microvasculopathy, and neuro-ophthalmic disorders..$^{21}$ However, if we understand the ocular manifestations by the more common RNA viruses which are known to cause a sequel of retinal changes and more commonly the retinitis. Komurasaki et al. ${ }^{22}$ reported the presence of vasculitis in the mice with the COVID 19.

The taxonomically diverse group of viruses may affect the eye as primary infection or reactivation. A recently published research by Marinha et.al. where eleven patients diagnosed with COVID $-19 .{ }^{23}$ These patients were known to show hyper-reflective lesions at the ganglion cell and inner plexiform layers levels. It has been shown more prominently at the papillomacular bundle in both eyes. ${ }^{23}$ Hence it becomes potent to undergo a retinal exam for each symptomatic or asymptomatic patient with COVID-19. ${ }^{19}$ The associated reduction in the cell arbitrated immunity makes the individual highly vulnerable to opportunistic viral ocular infections. This can be further explored by the speedily developing field of molecular diagnostics.

\section{PERSEPCTIVES}

The ocular diagnosis among COVID 19 patients is still in its infancy. The clinical diagnosis of the COVID 19 is done by assessing the body temperature, blood tests and RT PCR (reverse transcriptase-polymerase chain reaction) investigations. However, these diagnoses there still exist at risk and increase in the rate of transmission of the coronavirus.

There are many innovative kinds of research going on across the globe to cure this pandemic. As eye care professionals the knowledge and management strategies should be kept ready. A routine eye examination should be kept mandatory among such patients. The anterior segment evaluation with a slit lamp and a retinal eye exam using appropriate personal protective equipment is should be recommended. It can also be planned systematically to maintain proximity from patients and not to loosen up on the necessary investigations. Other than direct ophthalmoscopes, there are various other available tools that are quick in detecting the retinal findings and help to maintain proximity from the patient. Fundus imaging and the Optical Coherence Tomography (OCT) are a few procedures that can be done in a few clicks and are also non-contact. However, fundus fluorescence angiography (FFA) and indocyanine green (ICG) is the vasculature imaging techniques recommended if there are any signs of leakage in a fundus image or OCT.
Fundus images can capture the interior of the eye and remain an important imaging tool. This can be effectively achieved by digital fundus photography as well as confocal scanning laser ophthalmoscopy (cSLO). A fundus camera captures a definite one image of the fundus, on the other hand, cSLO uses a pinhole aperture through which laser light is projected and isolates only a single wavelength to capture an image. This may add false color to the images hence fundus photography through a camera captures the true color of the image. On the other hand, OCT has quickly become a mainstay for eye care professionals. OCT techniques are based on the principle of interferometry and are available in two different domains, namely, time-domain OCTs (TD-OCT), spectraldomain OCTs (SD-OCT), and recently developed frequencydomain OCTs (FD-OCT). Each of these domains is superior and differs from its predecessor in the net result as an enhancement in the resolution of images and wavelength-swept laser sources. Fundus imaging is a deep ocean for the eye care industry and innovation happens every now and then. There are various modalities and alternative techniques used for the diagnosis of retinal diseases and these have become a crucial part of an eye exam in today's pandemic scenario. ${ }^{24}$

\section{CONCLUSION}

Although existing knowledge about COVID-19 is rapidly growing. Evidence shows its transmission through tears; however, its risk may be truncated. This paper gives an insight into the ocular findings due to coronavirus. Also, it is proposed that retinal images may guide ophthalmologist to understand its effect on the retina. Imaging considering the anterior and posterior segment complications is possible, but the pathophysiology still remains unclear. This can be understood by extensive clinical research among COVID 19 patients. Further, these images along with artificial intelligence can provide a basis to automate the diagnosis of COVID 19. Eye care professionals should pool in resources and work together to understand these ocular manifestations and address the current pandemic in a scientific manner.

\section{Acknowledgement: Nil}

Competing interests: The authors declare no conflict of interest.

\section{Source of Funding: Nil}

\section{REFERENCES}

1. World Health Organization. WHO recommendations to reduce the risk of transmission of emerging pathogens from animals to humans in live animal markets. 2020. https://www.who.int/ health-topics/coronavirus/who-recommendations-to-reducerisk-of- transmission-of-emerging-pathogens-from-animals-tohumans-in- live-animal-markets. Accessed December 2020. 
2. Cheema M, Aghazadeh H, Nazarali S, Ting A, Hodges J, McFarlane A, et al. Kerato conjunctivitis as the initial medical presentation of the novel coronavirus disease 2019 (COVID-19): A case report. Can J Ophthalmol 2020;55(4):e125-e129.

3. Wang $\mathrm{D}, \mathrm{Hu} \mathrm{B}, \mathrm{Hu} \mathrm{C}$. Clinical characteristics of 138 hospitalized patients with 2019 novel coronavirus-infected pneumonia in Wuhan, China. J Am Med Assoc 2020;12:1-9.

4. Fang L, Karakiulakis G, Roth M. Are patients with hypertension and diabetes mellitus at increased risk for COVID-19 infection? Lancet Resp Med 2020;8(4):e21.

5. Otter JA, Donskey C, Yezli S, Douthwaite S, Goldenberg S, Weber DJ. Transmission of SARS and MERS coronaviruses and influenza virus in healthcare settings: the possible role of dry surface contamination. J Hospital Infect 2016;92(3):235-250.

6. Dowell SF, Simmerman JM, Erdman DD, Wu JS, Chaovavanich A, Javadi M, et al. Severe acute respiratory syndrome coronavirus on hospital surfaces. Clin Infect Dis 2004;39(5):652-657.

7. Qiao C, Zhang H, He M, Ying G, Chen C, Song Y, et al. Symptomatic COVID-19 in Eye Professionals in Wuhan, China. Ophthalmology 2020;127(9):1268-1270.

8. Turgut B. Role of ophthalmologists in combating with the Coronavirus disease 2019. Adv Ophthalmol Vis Syst 2020;10(2):3149.

9. Bacherini D, Biagini I, Lenzetti C, Virgili G, Rizzo S, Giansanti F. The COVID-19 pandemic from an ophthalmologist's perspective. Trends Mol Med 2020;26(6):529-531.

10. Huang C, Wang Y, Li X, Ren L, Zhao J, Hu Y, et al. Clinical features of patients infected with 2019 novel coronavirus in Wuhan, China. Lancet 2020;395(10223):497-506.

11. Ostler HB, Thygeson P. The ocular manifestations of herpes zoster, varicella, infectious mononucleosis, and cytomegalovirus disease. Survey Ophthalmol 1976;21(2):148-159.

12. Hong N, Yu W, Xia J, Shen Y, Yap M, Han W. Evaluation of ocular symptoms and tropism of SARS-CoV-2 in patients confirmed with COVID-19. Acta Ophthalmol 2020;10.1111/aos.14445.

13. Hamming I, Timens W, Bulthuis MLC, Lely AT, Navis GJ, van Goor H. Tissue distribution of ACE2 protein, the functional re- ceptor for SARS coronavirus. The first step in understanding SARS pathogenesis. J Pathol 2004;203(2):631-637.

14. Belser JA, Rota PA, Tumpey TM. Ocular tropism of respiratory viruses. Microbio Mol Bio Rev 2013;77(1):144-156.

15. Choudhary R, Kapoor MS, Singh A, Bodakhe SH. Therapeutic targets of renin-angiotensin system in ocular disorders. J Curr Ophthalmol 2017;29(1):7-16.

16. Seah I, Agrawal R. Can the coronavirus disease 2019 (COVID-19) affect the eyes? A review of coronaviruses and ocular implications in humans and animals. Ocular Immunol Inflam 2020;28(3):391-395.

17. Wu P, Duan F, Luo C, Liu Q, Qu X, Liang L, Wu K. Characteristics of ocular findings of patients with coronavirus disease 2019 (COVID-19) in Hubei Province, China. JAMA Ophthalmol 2020;138(5):575-578.

18. Bozkurt B, Eğrilmez S, Şengör T, Yıldırım Ö, İrkeç M. The COVID-19 Pandemic: Clinical Information for Ophthalmologists. Turk J Ophthalmol 2020;50(2):59.

19. American Academy of Ophthalmology. Alert: important coronavirus updates for ophthalmologists. www.aao.org/headline/alertimportant-coronavirus-context. Accessed on March 10, 2020.

20. Zhang X, Song W, Sun B, Mu J, Dong X, Wang B, et al. Conjunctival polymerase chain reaction-tests of 2019 novel coronavirus in patients in Shenyang, China. medRxiv 2020 Jan 1. doi: https://doi.org/10.1101/2020.02.23.20024935.

21. Newman H, Gooding C. Viral ocular manifestations: a broad overview. Rev Med Virol 2013;23(5):281-294.

22. Marinho PM, Marcos AA, Romano AC, Nascimento H, Belfort R. Retinal findings in patients with COVID-19. Lancet 2020;395(10237):1610.

23. Komurasaki Y, Nagineni CN, Wang Y, Hooks JJ. Virus RNA persists within the retina in coronavirus-induced retinopathy. Virology 1996;222(2):446-450.

24. Bernardes R, Serranho P, Lobo C. Digital ocular fundus imaging: a review. Ophthalmologica 2011;226(4):161-181. 